\title{
Effect of surface roughness on optical heating of metals
}

\author{
M. Auinger \\ michael.auinger.at@ieee.org \\ P. Ebbinghaus
}

\section{A. Blümich}

\section{A. Erbe}

a.erbe@mpie.de

\author{
Max-Planck-Institut für Eisenforschung GmbH, Max-Planck-Straße 1, 40237 Düsseldorf, Germany \\ Christian Doppler Laboratory for Diffusion and Segregation Phenomena during the Production of \\ High Strength Steel Sheets \\ Max-Planck-Institut für Eisenforschung GmbH, Max-Planck-Straße 1, 40237 Düsseldorf, Germany
}

Heating by absorption of light is a commonly used technique to ensure a fast temperature increase of metallic samples. The rate of heating when using optical heating depends critically on the absorption of light by a sample. Here, the reflection and scattering of light from UV to IR by surfaces with different roughness of iron-based alloy samples (Fe, $1 \mathrm{wt}-\% \mathrm{Cr}$ ) is investigated. A combination of ellipsometric and optical scattering measurements is used to derive a simplified parametrisation which can be used to obtain the absorption of light from random rough metal surfaces, as prepared through conventional grinding and polishing techniques. By modelling the ellipsometric data of the flattest sample, the pseudodielectric function of the base material is derived. Describing an increased roughness by a Maxwell-Garnett model does not yield a reflectivity which follows the experimentally observed sum of scattered and reflected intensities. Therefore, a simple approach is introduced, based on multiple reflections, where the number of reflections depends on the surface roughness. This approach describes the data well, and is subsequently used to estimate the fraction of absorbed energy. Using numerical modelling, the effect on the heating rate is investigated. A numerical example is analysed, which shows that slight changes in roughness may result in big differences of the energy input into a metallic sample, with consequences on the achieved temperatures. Though the model oversimplifies reality, it provides a physically intuitive approach to estimate trends.

[DOI: http://dx.doi.org/10.2971/jeos.2014.14004]

Keywords: Metals, infrared, scattering at rough surfaces, ellipsometry and polarimetry, mathematical methods (general), thermal emission

\section{INTRODUCTION}

The search for stable materials during fast temperature changes or thermal cycling conditions is one of the biggest challenges in modern alloy design. Therefore, a number of industrial and scientific research labs are investigating high temperature degradation properties of materials [1]-[3]. Apart from its industrial relevance (e.g. in steam power plants or combustion engines), fast temperature changes and well defined experimental conditions are extremely important to obtain reliable scientific results, which must not be influenced by reaction kinetics during heating or cooling. For many high temperature processes, optical heating has proven to be one of the best methods to achieve fast heating rates of more than $20 \mathrm{~K} \mathrm{~s}^{-1}$ with high accuracy $[4,5]$ and only little efforts for installation and maintenance.

Frequently, thermal light sources with an emission maximum in the near IR are used for heating purposes [6,5]. Light, impinging on a metallic substrate, may be absorbed (and contribute to heating), specularly reflected, or scattered. Following energy conservation, the sum of reflectivity $R$, absorption $A$, transmission $T r$ through the sample, and scattering $S$ should be unity, i.e.

$$
R+\operatorname{Tr}+A+S=1
$$

All quantities are considered normalised with respect to the incident intensity. For bulk metallic substrates, the transmission is zero $(\operatorname{Tr}=0)$ [7]. For efficient optical heating of a sample, $A=1-R-S$ should be maximised. While the reflectivity $R$ for a specularly reflected beam is essentially depending on the material of the substrate, light scattering $S$ strongly depends on surface properties, like roughness [8]-[12]. Despite the fact that optical heating is used in many modern hightemperature laboratories $[13,14]$, only little is known about the effects of surface preparation on efficiency of optical heating. Up to now, surface preparation is often chosen on an intuitive base. Often, there is a compromise between too rough (no uniform formation of corrosion products) and too smooth (uniform formation of corrosion products).

Recent years have seen a tremendous progress in the understanding of the optical properties of surfaces with nanoscopic up to microscopic roughness [15]-[17], surfaces decorated with or embedding small particles [12], [18]-[22], or regular, 
non-planar surfaces, including defects [11, 23, 24]. Nevertheless, a full, rigorous description of the scattering from an arbitrary metallic surface is still rather challenging. While full solutions of the Maxwell equations, e.g. by the finite element or finite difference method $[18,19,25,26]$, enables a full description of the intensity distribution after the scattering process, this procedure is rather tedious for surfaces with a complex internal structure of the roughness, as they are present in typical materials for high temperature applications.

Under the conditions of high temperature corrosion studies, an accurate determination of a sample's actual temperature is rather challenging. Soldering a temperature sensor to the metal surface is unsuitable for samples which should be subsequently subjected to surface analysis. Contact-less temperature measurements are easily affected by the IR light, used for heating. The most practical method therefore is the use of a reference sample, with an attached temperature sensor. Naturally, for reliable results about the high temperature behaviour of a sample, the heating behaviour of this reference needs to match the sample's behaviour as closely as possible.

It is the aim of this work to discuss the influence of surface roughness on the absorption $A$ of light by a metallic sample, and its consequences for heating. Therefore, this work shall contribute to allow direct comparisons between previously published works on high temperature processes. For this purpose, ellipsometric measurements over a large wavelength range from UV to IR are used to determine the pseudodielectric function of a Fe, $1 \mathrm{wt}-\% \mathrm{Cr}$ sample. Subsequently, interfacial properties, reflectivity and scattering from a series of $\mathrm{Fe}$, $1 \mathrm{wt}-\% \mathrm{Cr}$ samples with a systematically varied roughness are investigated by confocal microscopy, ellipsometry and scattering experiments. Aim of the optical characterisation is to evaluate the fraction of light, which is absorbed by estimating scattered and reflected fraction, for samples with different roughness. Because of the difficulties of a full physical description as needed for the solution of the Maxwell equations, a numerical model is introduced to estimate absorption of light (i.e. $A$ in Eq. (1)) over a large wavelength range on the basis of measurements of $R$ and $S$. Finally, the heating of a sample is analytically evaluated and numerically explored on the basis of the sample's absorption efficiency.

\section{EXPERIMENTAL}

Cast alloy samples of $\mathrm{Fe}, 1 \mathrm{wt}-\% \mathrm{Cr}$ (in-house production) were cut into square shaped pieces with dimensions $15 \mathrm{~mm} \times 15 \mathrm{~mm} \times 2 \mathrm{~mm}$. The sample surfaces were prepared with different roughness by applying a preparation sequence, starting from mechanical grinding with grinding papers of 400 grit size down to 4000 grit, followed by polishing steps with different diamond pastes $(6 \mu \mathrm{m}, 3 \mu \mathrm{m}, 1 \mu \mathrm{m})$ and a surface finish with $100 \mathrm{~nm}$ silica suspension. This sequence was stopped for each individual sample, as soon as the desired surface preparation was reached. Finally, all samples were ultrasonically cleaned in ethanol and dried in a cold stream of dry air.

Surface topographies and mean roughness values were deter- mined with the help of a confocal microscope ( $\mu$ Surf Explorer, NanoFocus Messtechnik GmbH, Germany) by measuring areas of $320 \mu \mathrm{m} \times 320 \mu \mathrm{m}$ and $500 \mu \mathrm{m} \times 500 \mu \mathrm{m}$ to compare statistical deviations. This technique allows a contact-free determination of the surface roughness, which minimises influences for subsequent optical experiments.

Surface light scattering experiments were carried out on a modified single wavelength ellipsometer (Multiskop, Optrel GbR, Germany) at a wavelength of $532 \mathrm{~nm}$ for three different angles of incidence $\alpha\left(30^{\circ}, 45^{\circ}\right.$ and $\left.70^{\circ}\right)$. The detection angle $\alpha^{\prime}$ has been varied from $25^{\circ}$ to $85^{\circ}$. The intensity, scattered into a solid angle of $6.90 \mathrm{msr} \approx \pi / 455 \mathrm{sr}$ around the scattering angle $\theta=\alpha^{\prime}-\alpha$, has been detected by an optical power meter (NOVA II, Ophir Optics, Israel) and normalised to the incident intensity, which was measured before the experiment. Also, incident and analysed polarisation have been changed between parallel (p) and perpendicular (s) polarisations in the absence of a quarter wave plate. In each experiment, only scattering vector components within the scattering plane (defined by the wave vector of the incident beam and the surface normal vector of the sample) were analysed. After the first measurement of a sample, the sample was rotated by $90^{\circ}$ and measured again to account for in-plane anisotropy in surface preparation. Optical characterisation of the wavelengthdependence of the optical properties was conducted by spectroscopic ellipsometry. In the range from $300 \mathrm{~nm}-820 \mathrm{~nm}$, all samples were measured on a SE 800 (Sentech Instruments, Germany) spectroscopic ellipsometer. Mid-IR measurements in the spectral range from $2.5 \mu \mathrm{m}$ to $25 \mu \mathrm{m}$ were conducted using a SENDIRA (Sentech Instruments, Gemany) IR spectroscopic ellipsometer connected to a Biorad FTS-3000 FTIR spectrometer (Sentech Instruments, Germany). For the sample which was polished to the flattest surface finish, the full pseudodielectric function was determined on the basis of a measurement in the range from $200 \mathrm{~nm}-1700 \mathrm{~nm}$ on a SE 850 DUV (Sentech Instruments, Germany).

\section{RESULTS AND DISCUSSION}

\subsection{Surface Roughness}

Topography of the ground samples (Figure 1) showed a strong anisotropy, as the specimens were not rotated during grinding. The polished sample surfaces appeared to be homogeneous due to additional counter-clockwise rotation of the specimen on the preparation disk, which meets standard sample preparation techniques [27].

As shown in Table 1, the root mean square roughness values $S_{\mathrm{q}}$ strongly depend on the orientation of the samples but decrease with continuation of the grinding and polishing procedure. A big decrease of the mean area roughness value can be observed in the case of grinding with 1000 grit, 2500 grit and 4000 grit size papers. These three grit sizes are the most frequently used surface preparations for high temperature corrosion testing. However, they span a large range of roughness values and hence leads to poorly comparable results between different corrosion studies. 


\begin{tabular}{ccccc}
\hline surface treatment & $\begin{array}{c}S_{\mathrm{q}}^{\text {(hor) }} / \\
\mu \mathrm{m}\end{array}$ & $\begin{array}{c}S_{\mathrm{q}}^{\text {(vert) }} \\
\mu \mathrm{m}\end{array}$ & $\begin{array}{c}S_{\mathrm{q}}^{\text {(area) }} \\
\mu \mathrm{m}\end{array}$ & $\begin{array}{c}f_{\mathrm{sp}}^{\text {(area) }} \\
\mu \mathrm{m}^{-1}\end{array}$ \\
\hline 400 grit & 0.094 & 0.304 & 0.318 & $0.05-1.65$ \\
600 grit & 0.055 & 0.241 & 0.247 & $0.05-1.50$ \\
1000 grit & 0.044 & 0.248 & 0.252 & $0.05-2.00$ \\
2500 grit & 0.022 & 0.105 & 0.107 & $0.05-2.75$ \\
4000 grit & 0.024 & 0.027 & 0.036 & $0.05-4.30$ \\
$6 \mu \mathrm{m}$ paste & 0.020 & 0.029 & 0.035 & $0.05-2.80$ \\
$3 \mu \mathrm{m}$ paste & 0.028 & 0.029 & 0.040 & $0.05-1.25$ \\
$1 \mu \mathrm{m}$ paste & 0.018 & 0.019 & 0.026 & $0.05-0.25$ \\
100 nm paste & 0.006 & 0.006 & 0.008 & $*$ \\
\hline \multicolumn{4}{c}{ * values too small for the scanned frequency range }
\end{tabular}

TABLE 1 Average root mean square roughness $S_{\mathrm{q}}$ and spatial frequency range $f_{\mathrm{sp}}^{(\text {area })}$ of Fe, 1 wt- $\% \mathrm{Cr}$ alloys with different surface finish ( $S_{\mathrm{q}}^{(\text {hor })}$ : parallel to grinding direction, $\mathrm{s}_{\mathrm{q}}^{(\text {vert })}$ : perpendicular to grinding direction, $\mathrm{s}_{\mathrm{q}}^{(\text {area })}$ : entire surface).
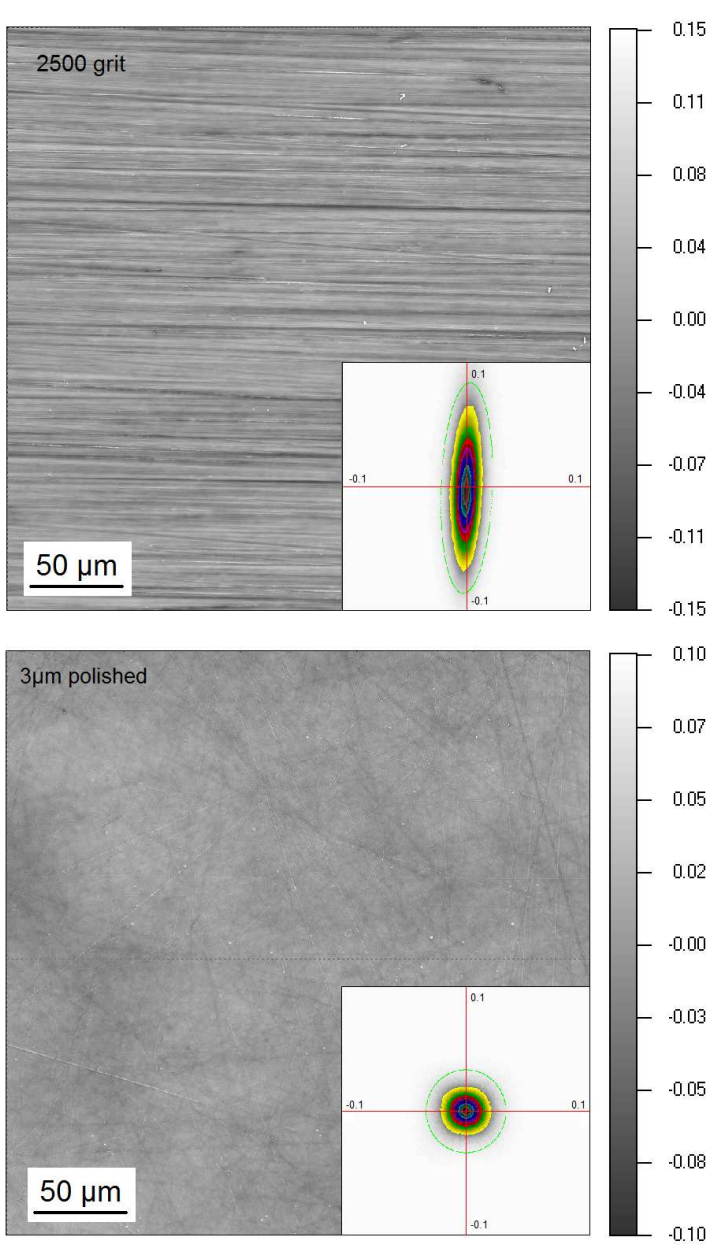

FIG. 1 Confocal microscopy images of the surface topography of $\mathrm{Fe}, 1 \mathrm{wt}-\% \mathrm{Cr}$ alloys with different surface finish. The inset shows the angular dependency of the roughness value $S_{q}$. Note the difference in the height scales.

Fast Fourier Transform analyses of the averaged horizontal and vertical surface profiles have been performed to identify the main bandwidth of the spatial frequencies $f_{\mathrm{sp}}$, that compose the surface structure. The spatial frequency range expands with finer grit size papers, caused by finer grooves during the grinding process. For the polished samples, the opposite trend could be observed. This originates from the additional counter-clockwise rotation on the preparation disk, preventing strong anisotropies of the surface pattern. The con-
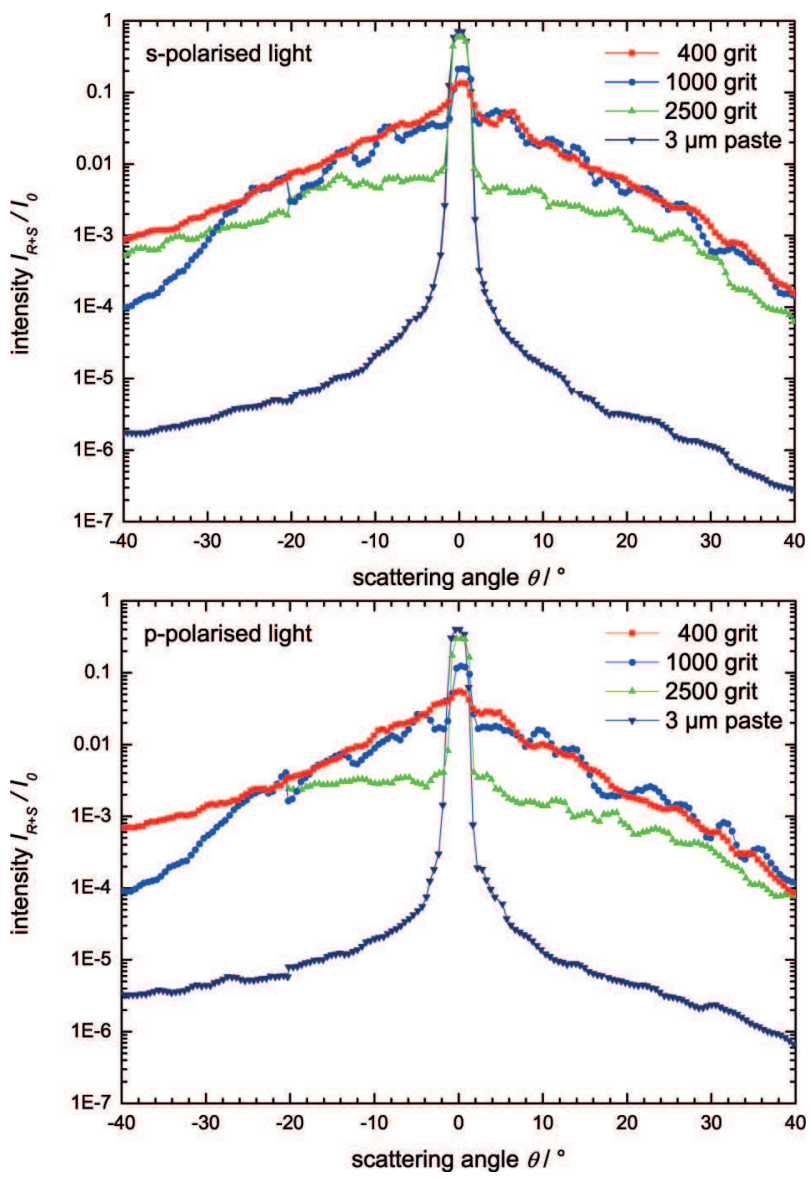

FIG. 2 Measured optical scattering of s-polarised and p-polarised light $(\lambda=532 \mathrm{~nm}$, $\alpha=45^{\circ}$ ) on $\mathrm{Fe}, 1 \mathrm{wt}-\% \mathrm{Cr}$ alloys with different surface finish as indicated on the graph.

sidered range of the spatial frequencies for all cases was 0.05 $10 \mathrm{\mu m}^{-1}$, corresponding to a periodicity of the surface patterns between $20 \mu \mathrm{m}$ and $100 \mathrm{~nm}$.

\subsection{Reflection and Scattering Measurements}

Figure 2 shows the intensity distribution of the reflected and scattered light on $\mathrm{Fe}, 1 \mathrm{wt}-\% \mathrm{Cr}$ samples with different surface treatments. A clear difference in the scattering behaviour with sample roughness could be verified for both, s-polarised and p-polarised light. The biggest change in the scattering be- 

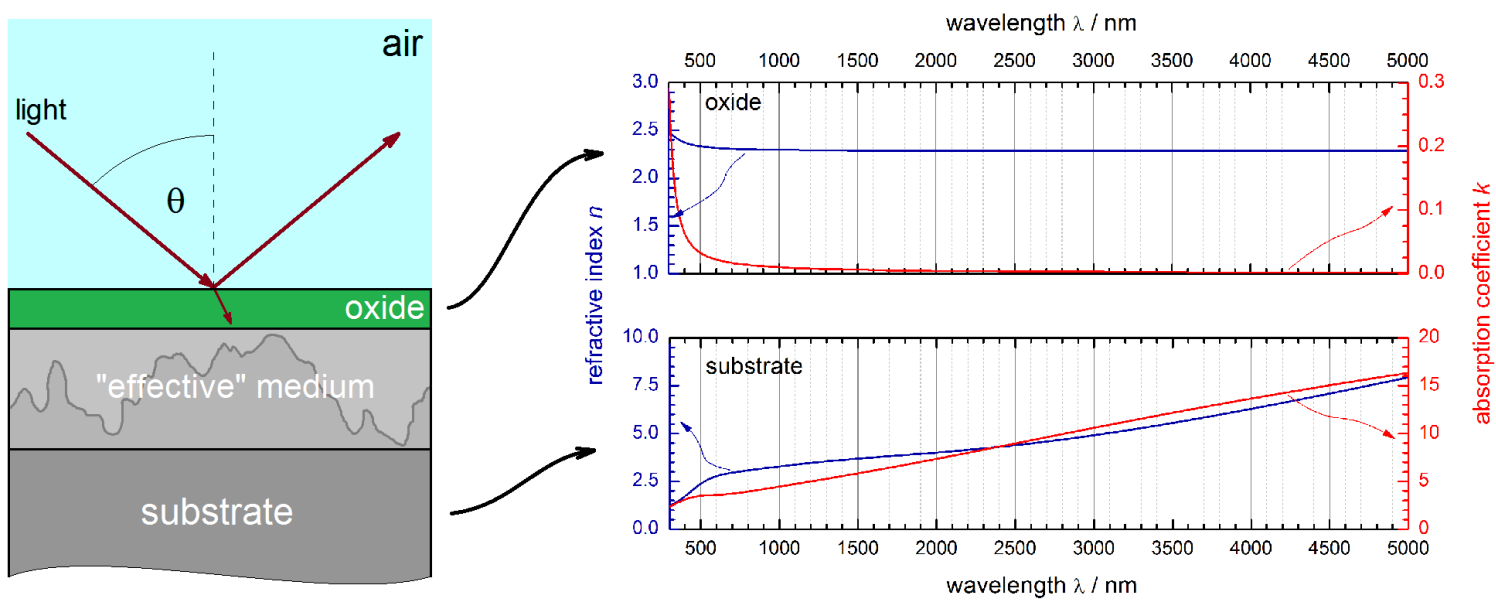

FIG. 3 Schematic picture of the model, used for ellipsometry data analysis (left) and optical properties of both substrate and oxide layer (right). Optical properties of the effective medium layer are defined by the optical properties of substrate with inclusions of air via the Maxwell-Garnett equation.

haviour occurred between 1000 grit and 2500 grit size grinding papers, where the intensity of the specularly reflected beam $\left(0^{\circ}\right)$ changes by almost a factor of three. Measurements between crossed polarisers were conducted as well, but intensities have been found only slightly above the extinction ratio of the crossed polarisers.

Ellipsometry data were fitted by a multilayer model [28]-[30], shown in Figure 3. Initially, data from the flattest sample were fitted with a three layer model including ambient - oxide film - substrate. The dielectric function of the Fe, 1wt- $\%$ Cr substrate was modelled by a Drude-Lorentz model. In addition, the effect of a thin oxide layer was taken into account by using a single Lorentzian oscillator with a maximum frequency in the UV. These data served as input for the modelling of data from the samples with varying roughness and is listed in Appendix A, together with the equations used in the analysis. For systematic variation of the roughness, a substrate with the $\mathrm{Fe}, 1 \mathrm{wt}-\% \mathrm{Cr}$ dielectric function was considered, and on the substrate a layer to account for the roughness (Figure 3). This layer was described by an effective medium model [28] on the basis of the Maxwell-Garnett equation. Both the thickness of the layer and the substrate / air ratio were allowed to vary in the modelling. The top layer represents the optical properties of a native oxide layer, as obtained from the smoothest specimen. Its thickness was modelled as $1.20 \mathrm{~nm}$ and has not been varied for all other samples, as they were all prepared under the same conditions. In all analyses, an ambient air with a refractive index of 1 was considered.

Simulation results indicate a strong dependency of the effective medium thickness on the corresponding roughness of the substrate for high $S_{\mathrm{q}}$-values (Figure 4). A clear decrease of the effective medium thickness $d_{1}$ and no major change of the fraction of air $f_{\text {Air }}$ can be observed, as the surface preparation has been continued with finer grinding papers. It should be mentioned that effective medium thickness $d_{1}$ and the volume fraction of air $f_{\text {Air }}$ are partially correlated, especially in cases of smoother samples. They are coupled in a way that the layer thickness will increase with an increase of the fraction of air in

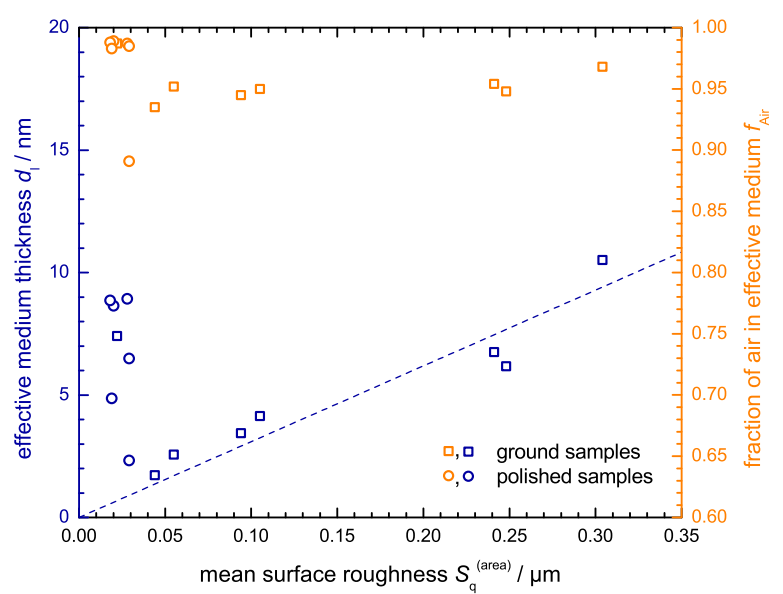

FIG. 4 Dependence of the thickness and substrate / air ratio of the effective medium layer with respect to the sample roughness for ground samples ( $\square$ ) and polished samples (o). The dashed line was derived from a linear fit of the ground samples, except for the smoothest surface.

the effective medium to still yield similar optical properties of the layer.

High thickness values of the effective medium may additionally depend on the surface preparation technique, which may cause strong anisotropies of the surface topography [7, 15]. The fraction of air in the effective medium is considerably higher for the polished samples, compared to the ground specimens. Consequently, this yields higher values for the effective medium layer thickness. To account for this behaviour, obtained fit results were divided into two different groups ground samples and polished samples.

On the basis of the results of the fits of the ellipsometric data, reflectivities in s- $\left(R_{\mathrm{s}}\right)$ and p-polarisation $\left(R_{\mathrm{p}}\right)$ have been calculated, using the same models as for fitting, and averaged to obtain the reflectivity of unpolarised light $R=\frac{1}{2}\left(R_{\mathrm{p}}+R_{\mathrm{s}}\right)$. 
These calculated reflectivities at a certain wavelength have been compared to the experimentally determined sum of normalised reflected and scattered intensity, $R+S$ (Figure 5(a)). As is to be expected, there is a difference between calculated $R$ and experimental $R+S$, which increases with increasing sample roughness. The underlying Fresnel model $[29,30]$ for the ellipsometry data only considers reflections on ideally flat interfaces between individual layers and therefore cannot accurately account for the effects of scattering. Scattering is, however, present in the actual samples, as the measurements show.

Systematic investigation of reflection and scattering [32] have been performed for sinusoidal-shaped surface morphologies [24], other well-defined geometries [11, 15, 16, 17, 23, 33] and sub-surface defects [21, 34]. Simplified models exist also for special cases, where the optical wavelength is much smaller (or much larger) than the in-plane periodicity of a surface pattern [35]. A description of random roughness over different length scales is still in its infancy. In all possible models, some knowledge is needed about details of the surface topography. However, the obtained surfaces from grinding and polishing do not possess simple topographies, and contain roughness over many different length scales.

Therefore, the samples investigated here will be treated in a geometric optics approach. On rough samples with roughness on length scales bigger than the wavelength, a beam of light that is reflected once can undergo $N-1$ further reflections, from other parts of the surface. While this description is not fully physically correct for roughness in the order to the wavelength, the phenomenon of multiple scattering from a rough sample would result in a very similar behaviour, which can be effectively described by the same numerical model as describing multiple reflection in geometrical optics. Using the reflectivity $R$ obtained from modelling ellipsometric measurements from an ideally flat sample, one can then obtain the intensity $I_{R+S}$ of scattered and reflected light from a rough sample as

$$
I_{R+S}=I_{0} R^{N}=I_{\mathrm{ref}} R^{N-1} .
$$

Here, the sample with the smallest roughness, which scatters and reflects the intensity $I_{\text {ref }}$ at an incident intensity of $I_{0}$, is treated approximately as ideally flat.

Eq. (2) is now used to relate all measured $(R+S)$ to measurements of the flattest sample. Good agreement with experimental observations could be verified for all investigated specimens (Figure 5(a)), with an average number of reflection events $N$ ranging from 1 (smooth surface) to 7 (rough surface) at $\alpha=45^{\circ}$. For an extension of the description to different $S_{\mathrm{q}}$ and $\alpha$, a polynomial fit function, with parameters $a$ and $b$,

$$
\begin{aligned}
N\left(S_{\mathrm{q}}, \alpha\right)= & 1+a \cdot \cos (\alpha)\left(S_{\mathrm{q}}-S_{\mathrm{q}, \mathrm{ref}}\right) \\
& +b \cdot \cos (\alpha)\left(S_{\mathrm{q}}-S_{\mathrm{q}, \mathrm{ref}}\right)^{2},
\end{aligned}
$$

has been introduced by using the restriction of a single reflection both at the roughness value of the flattest sample $\left(S_{\mathrm{q}, \text { ref }}=S_{\mathrm{q}}^{\text {(area) }}=0.008 \mu \mathrm{m}\right)$ as well as for $\alpha=90^{\circ}$. For better comparison, the graphical representation of the correlation between $N, S_{\mathrm{q}}$ and $\alpha$ is shown in Figures 9 and 10 in Appendix A.
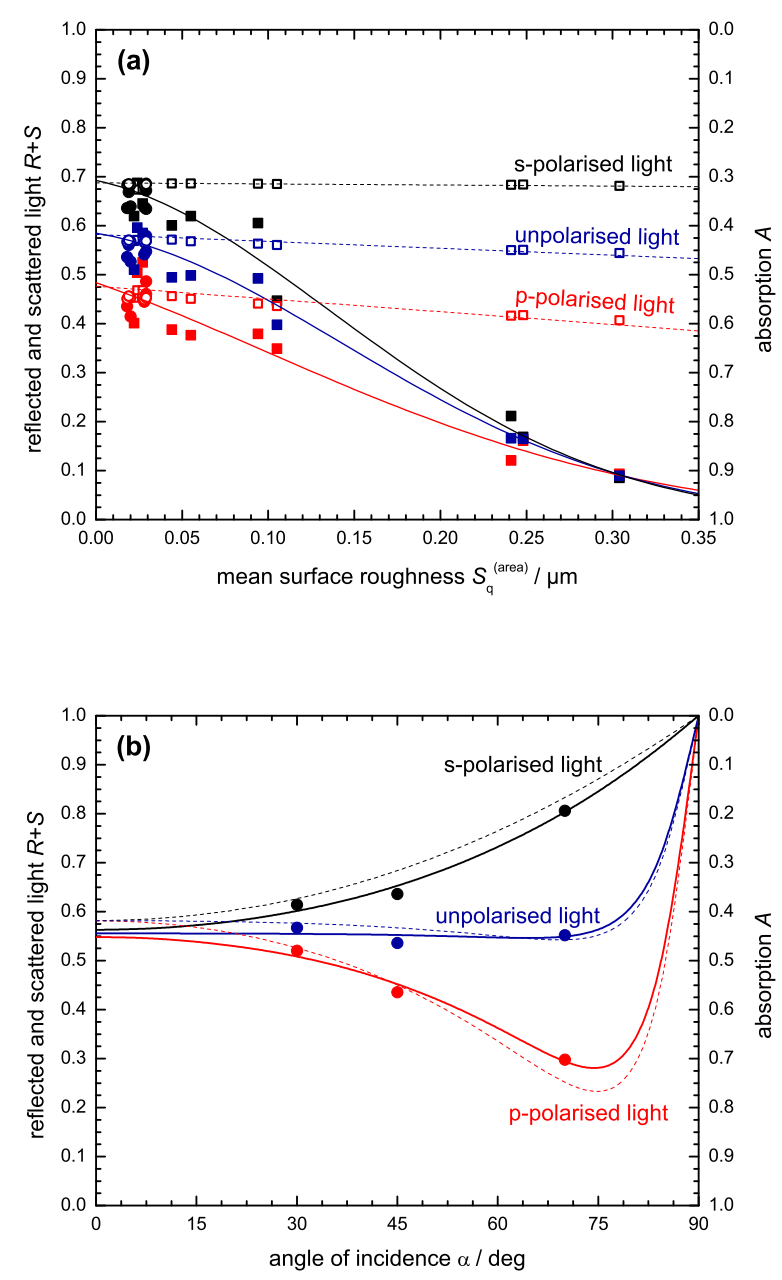

FIG. 5 Sum of reflectivity and scattering contribution $R+S(\lambda=532 \mathrm{~nm})$ of Fe, $1 \mathrm{wt}-\% \mathrm{Cr}$ surfaces as function of the sample roughness at $\alpha=45^{\circ}$ (left). $R+S$ as function of angle of incidence for a surface, polished with $1 \mu \mathrm{m}$ suspension $\left(S_{\mathrm{q}}^{(\text {area })}=0.026 \mu \mathrm{m}\right.$, right). Hollow symbols represent results, calculated on the basis of the fitted ellipsometry data ( $R$ only, $S=0$ ). Filled symbols correspond to experimental results, integrating the reflected and scattered intensities $(R+S)$ for ground samples $(\square, \boldsymbol{\square})$ and polished samples $(\circ, \bullet)$. Dotted lines represent the behaviour of the model, used to fit the ellipsometric data. The solid lines were derived from the numerical description of the roughness dependence in Eqs. (2) and (3).

The obtained experimental values of $R+S$ in s- and ppolarisation as well as for unpolarised light are shown in Figure 5(b), together with results of the numerical description. The experimental points are close to the expected values from the numerical description. The angular dependence of $R+S$ for a surface treated with $1 \mu \mathrm{m}$ suspension shows a small, but systematic, deviation from expected values. At grazing incidence $\left(\alpha=90^{\circ}\right)$ one expects $R+S=1$. Slight deviations between data derived on the basis of ellipsometric measurements and those derived from the numerical description are found at low $\alpha$, especially for p-polarised light. However, the numerical description nicely captures the roughness dependence of $R+S$.

Experimental results for $S$ have only been derived at $\lambda=532 \mathrm{~nm}$. However, in the description, the wavelengthdependence is fully included in the dielectric function of the 

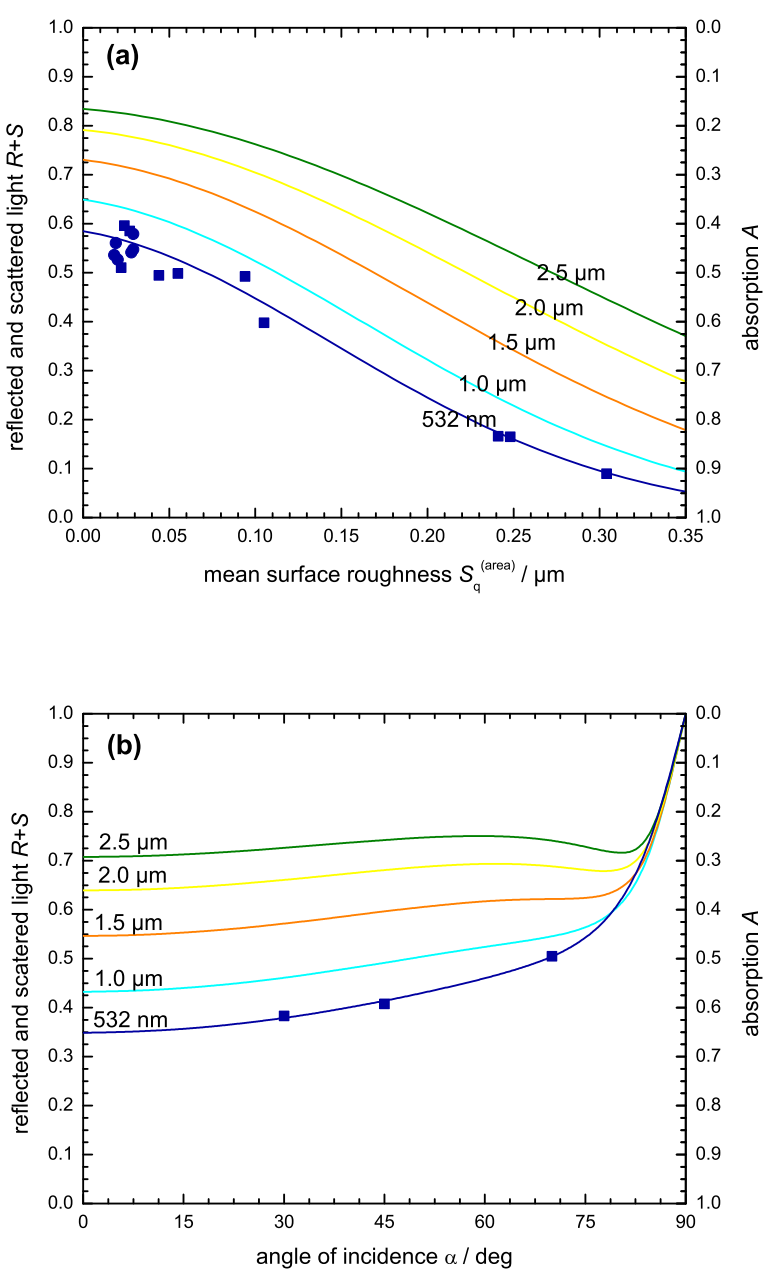

FIG. 6 Light absorption derived from Eqs. (2) and (3) as function of $S_{\mathrm{q}}^{(\text {area) }}$ at $\alpha=45^{\circ}$ (a) and as function of $\alpha$ for a Fe, $1 \mathrm{wt}-\% \mathrm{Cr}$ surface prepared with 2500 grit size grinding paper with $S_{\mathrm{q}}^{(\text {area })}=0.107 \mu \mathrm{m}$ (b). All data are presented at different wavelengths.

substrate. For optical heating, the wavelengths of interest is $800 \mathrm{~nm}-2.5 \mu \mathrm{m}[36,37]$, an extension to all wavelengths was performed. For this purpose, $N$ was considered independent of $\lambda$. At the same time, Eq. (1) was invoked to estimate $A$ for different $\lambda$ and $\alpha$ (Figure 6). As expected, for all $\lambda, A$ decreases with decreasing $S_{\mathrm{q}}$ (Figure 6(a)). Furthermore, $R+S$ increases with increasing $\lambda$ within the entire roughness range. This increase becomes even more pronounced for rough surfaces (high $S_{\mathrm{q}}$ ). From Figure 6 it becomes clear that the absorbed light and hence optical heating drastically increases with sample roughness. When comparing $S_{\mathrm{q}}$ with their corresponding surface treatment (Table 1), the biggest drop of observed intensity occurs between 1000 grit and 2500 grit sized grinding papers. $R+S$ for a 2500 grit size treated surface shows a very strong dependency with $\alpha$ (Figure 6(b)).

When comparing the obtained results for rough samples with the results for smoother surfaces (Figure 5(b)) it becomes clear that $R+S$ may change by more than $20 \%$ for the same $\alpha$. This implies a strong correlation of the angular dependency of $S$ from $S_{\mathrm{q}}$. A graphical representation of all parameters of the numerical description and the statistical fit can be found in Figure 9 in Appendix A. (a)
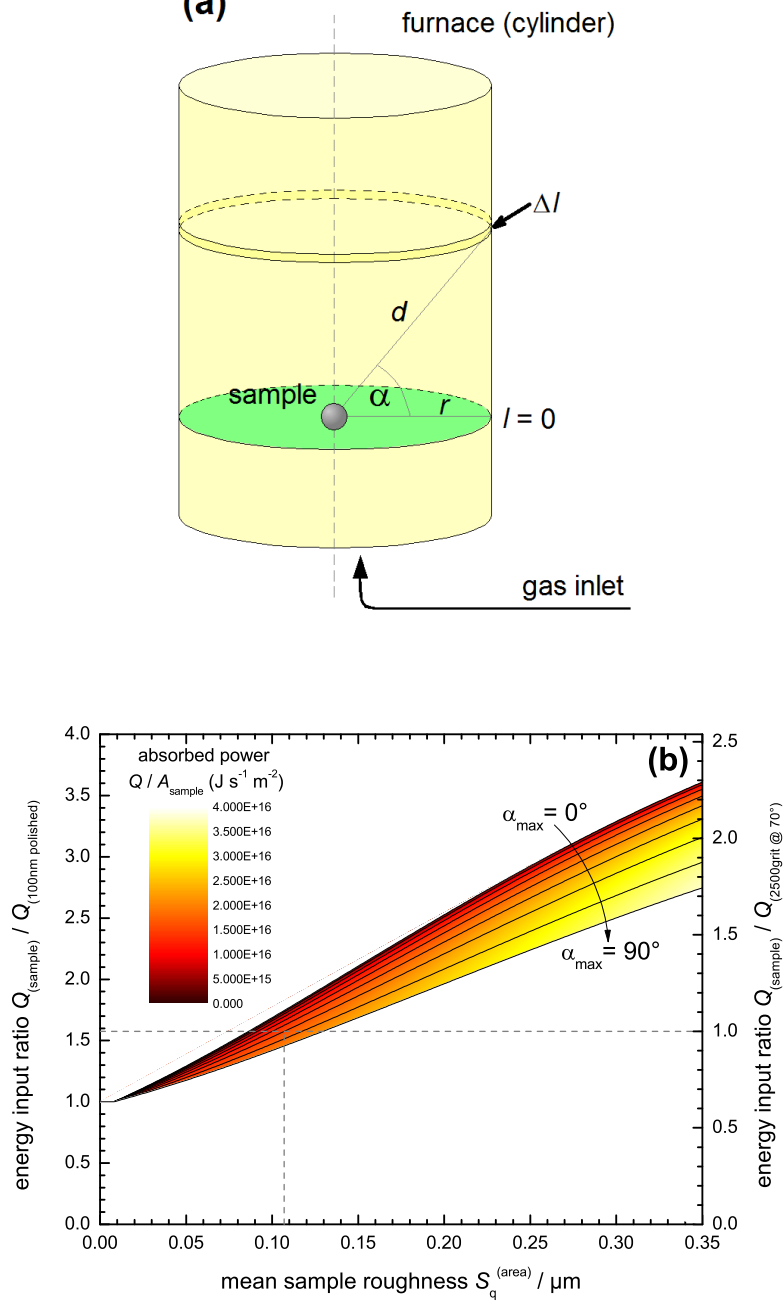

FIG. 7 Scheme of a sample in an optical heating furnace (a). Calculated optical heating properties, depending on the sample roughness (b). Note the two different axes in (b), which apply to two different reference samples (100 nm polished and 2500 grit ground).

\subsection{Consequences for Optical Heating}

To obtain a valid estimate for the optical heating behaviour by classical physical models without discussing geometrical issues of oven construction, it is assumed that the sample of interest is small and surrounded by a homogeneously radiating cylinder which represents the furnace (Figure 7(a)). Furthermore, the optical emission spectrum of the furnace has been modelled by the emission characteristics of an ideal black body emitter at $1600^{\circ} \mathrm{C}$, possessing its maximum intensity at $\lambda=1.55 \mu \mathrm{m}$.

Since the calculation of the total absorbed energy and the actual temperature change of the sample is a quite long mathematical procedure [39], only the essence of obtained results will be shown in this paragraph. A detailed mathematical model with all important formulae to describe optical heating efficiency is included in Appendix B.

As obvious from Figure 7(b), the absorbed light intensity significantly increases for higher maximum angle of incidence $\alpha_{\max }$ (defines the cylinder's length), which corresponds to an increase of the furnace length. The ratio between energy into 
sample and into the reference (which is used to monitor the temperature inside the device) however, tends to decrease as the differences of absorbed energies are getting smaller. Here, choosing the smoothest sample (100 nm polished surface, $S_{\mathrm{q}}^{(\text {area) }}=0.008 \mu \mathrm{m}$ ) as a reference for the energy input ratio originates from previous definitions for the number of reflection events (Eq. (2)) and not from practical applications. From an experimental point of view, the results in Figure 7(b) show that the reference needs to match the surface preparation of the sample as closely as possible because the energy input ratio can be up to 3 times higher for rough samples (meaning that these samples absorb three times the energy than the reference). Therefore, a second axis for the energy input ratio has been inserted in Figure 7(b), which normalises to the roughness of a 2500 grit ground sample $\left(S_{\mathrm{q}}^{(\text {area })}=0.107 \mu \mathrm{m}\right)$.

A simple calculation of the actual sample temperature has been based on energy conservation assuming that all absorbed light contributes to heating only of the sample, which stands in thermal equilibrium with the gas stream that controls the atmosphere over the sample. Besides the flow of gas, thermal radiation of a hot sample is a second loss channel [38]. It will be assumed that the energy input ratio $f$ as well as the ratio of the heat capacities from the sample and the gas do not change with temperature. Variations in the thickness of surface oxides (e.g. the iron and/or chromium oxides in this study) may cause additional deviations [8]-[11], [17, 20, 40], if they should be present at elevated temperatures and atmospheric conditions of interest. Furthermore, the sample and the reference are assumed to be similar in mass and composition. Energy conservation implies for the reference

$$
\dot{Q}_{\mathrm{ref}}=m_{\mathrm{Fe}} c_{\mathrm{p}, \mathrm{Fe}} \frac{\mathrm{d} T}{\mathrm{~d} t}+\dot{n}_{\mathrm{gas}} \mathcal{C}_{\mathrm{p}, \mathrm{gas}}\left(T-T_{\mathrm{in}}\right)+\epsilon \sigma A_{\mathrm{smp}} T^{4},
$$

and for the sample

$$
\begin{aligned}
\dot{Q}_{\mathrm{smp}} & =f \dot{Q}_{\mathrm{ref}} \\
& =m_{\mathrm{Fe}} \mathcal{c}_{\mathrm{p}, \mathrm{Fe}} \frac{\mathrm{d} \tilde{T}}{\mathrm{~d} t}+\dot{n}_{\text {gas }} c_{\mathrm{p}, \text { gas }}\left(\tilde{T}-T_{\mathrm{in}}\right)+\epsilon \sigma A_{\mathrm{smp}} \tilde{T}^{4} .
\end{aligned}
$$

The meaning of the symbols is given in Table 2 .
An analytical solution for the general case of Eqs. (4) and (5) the actual sample temperature by applying an arbitrary heating programme - can not be given. The radiation energy term and the time-dependent energy input characterise an inhomogeneous Bernoulli type ordinary differential Eq. [41] which can not be solved explicitly. However, the gradient field of this equation indicates a stable and well-behaved solution in the usual temperature (and time) range, which allows for numerical solutions of these equations.

At low temperatures, the radiation term becomes negligibly small [38], which even allows for an analytical solution of the simplified case by considering just sample and gas heating as

$$
\begin{aligned}
\tilde{T}_{(t)}= & f T_{(t)}+(1-f) T_{\text {in }}+(1-f)\left(T_{0}-T_{\text {in }}\right) \\
& \cdot \exp \left(-\frac{\dot{n}_{\text {gas }} c_{\mathrm{p}, \text { gas }}}{m_{\mathrm{Fe}} c_{\mathrm{p}, \mathrm{Fe}}} t\right) .
\end{aligned}
$$

Eq. (6) shows the actual sample temperature with a fixed settemperature programme for the reference. As the energy input ratio $f$ changes with sample roughness, serious temperature differences between reference and sample could occur. The missing term for the energy loss due to optical radiation of the sample leads to a significant change of temperature above $300^{\circ} \mathrm{C}$ and reduces the overheating of the samples (Figure 8). Detailed mathematical descriptions of the temperature difference during optical heating can be found in Appendix C.

A graphical representation of the numerical results for the case of illumination with constant light intensity (Figure 8(a)) and by applying a heating program, composed of a linear heating rate, followed by isothermal exposure and cooling in the ambient gas stream (Figure $8(\mathrm{~b})$ ) is shown. It can be clearly seen that only minor differences of the sample roughness lead to a temperature difference between sample and reference, exceeding $100^{\circ} \mathrm{C}$. Consequently, erroneous results may be ob-

\begin{tabular}{|c|c|}
\hline$T$ & temperature of reference; set-temperature \\
\hline$\tilde{T}$ & temperature of the sample \\
\hline$T_{\text {in }}$ & temperature of the gas at the inlet \\
\hline$\dot{Q}$ & absorbed optical energy from cylinder \\
\hline$f$ & energy input ratio \\
\hline$m_{\mathrm{Fe}}$ & mass of the sample / reference \\
\hline$c_{\mathrm{p}, \mathrm{Fe}}$ & $\begin{array}{l}\text { specific heat capacity of sample / reference } \\
\text { (example here: Fe, } 0.449 \mathrm{~J} \mathrm{~g}^{-1} \mathrm{~K}^{-1} \text { [42]) }\end{array}$ \\
\hline$\dot{n}$ & $\begin{array}{l}\text { molar flux of the gas stream } \\
\text { (example here: } 2.479 \cdot 10^{-4} \mathrm{~mol} \mathrm{~s}^{-1}, 201 \mathrm{~h}^{-1} \text { ) }\end{array}$ \\
\hline$c_{\mathrm{p}, \text { gas }}$ & $\begin{array}{l}\text { molar heat capacity of the gas } \\
\text { (example here: argon, } 20.786 \mathrm{~J} \mathrm{~mol}^{-1} \mathrm{~K}^{-1} \text { [42]) }\end{array}$ \\
\hline$\epsilon$ & emissivity (example here: Fe, 0.15 [43]) \\
\hline$\sigma$ & Stefan-Boltzmann's constant \\
\hline$A_{\text {smp }}$ & $\begin{array}{l}\text { sample surface (example here: } 2 \cdot 10^{-4} \mathrm{~m}^{2} \\
\text { for a } 1 \mathrm{~cm}^{2} \text { sized specimen) }\end{array}$ \\
\hline
\end{tabular}
tained when surface preparation of sample and reference are different, especially when investigations are carried out near a phase transformation temperature of the sample (e.g. the ferrite - austenite transition of iron). 

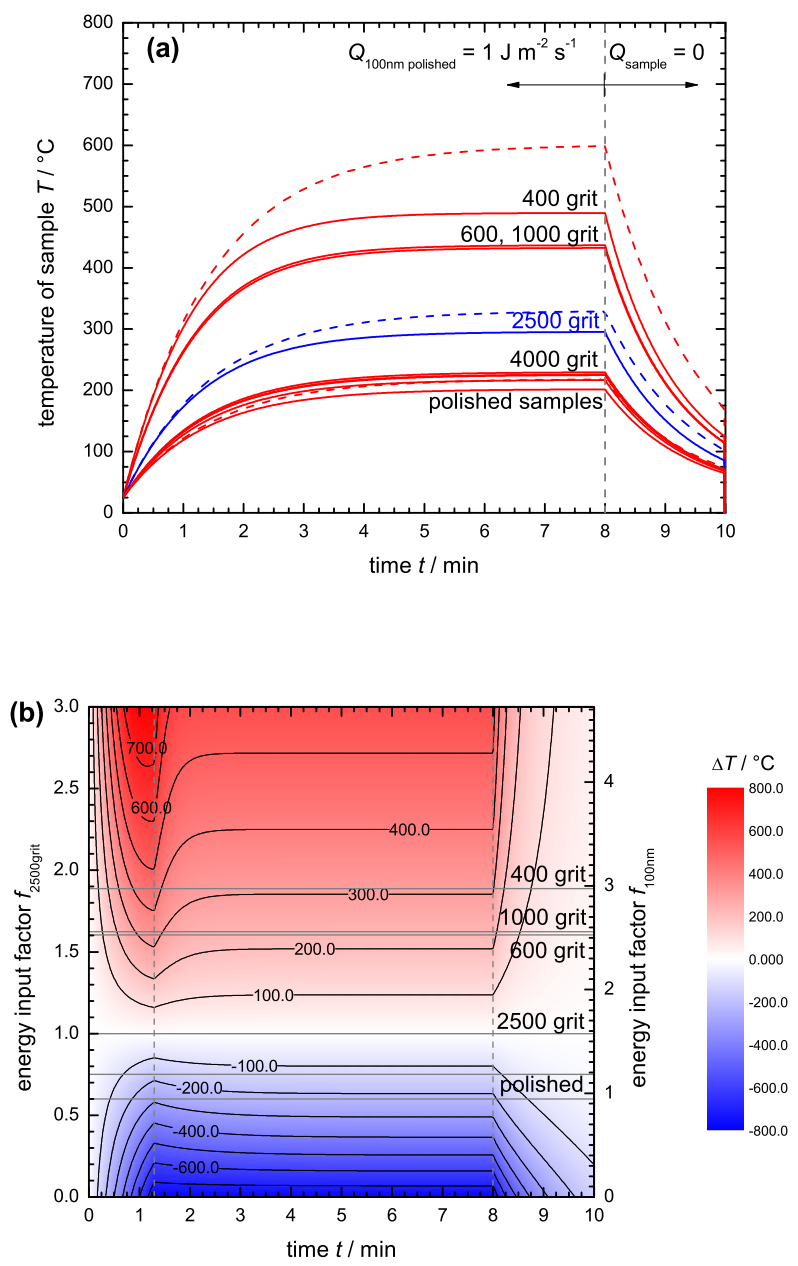

FIG. 8 Calculated temperature evolution in $\mathrm{Fe}, 1 \mathrm{wt}-\% \mathrm{Cr}$ samples with different surface roughness, by applying a constant optical energy $\dot{Q}_{\text {ref }}$ (a) and by linear heating with $10 \mathrm{~K} \mathrm{~S}^{-1}$ up to $800^{\circ} \mathrm{C}$, isothermal dwelling for $6.5 \mathrm{~min}$ and cooling in the flowing gas stream (b). Dashed lines in (a) represent the analytical solution for the temperature without heat radiation (Eq. (6)). All simulations were calculated for a furnace with $\alpha_{\max }=70^{\circ}$

\section{CONCLUSION}

Optical heating is a state-of-the-art heating method which allows extremely fast temperature changes in metallic samples [44]. The most common method to evaluate the temperature of the sample under investigation is the use of a reference of the same material. The results here show clearly, that sample surface preparation of the reference needs to match the preparation of the sample for reliable temperature measurements.

Clear evidence of the relation between surface roughness, angles of incidence and optical absorption properties (and consequently sample heating) could be established. Although reflectivity strongly depends on the actual surface topography (wavy structures, spikes, cracks, pits, defects, ...) and not only on roughness values alone [7, 10, 12, 15, 16, 19, 32, 33, 35, 45], it needs to be mentioned that samples with a mean surface roughness lower than $0.05 \mu \mathrm{m}$ or incidence angles $\alpha>75^{\circ}$ are strong reflectors of incident light, which results in extreme difficulties for optical heating. This roughness limit cor- responds to a surface treatment with 4000 grit size grinding paper or a subsequent polishing step. A surface roughness above $0.20 \mu \mathrm{m}$ possesses a remarkable increase in optical absorption (increasing at $1.5 \mu \mathrm{m}$ by almost a factor of 3 ) leading to a higher efficiency for optical heating. However, the measured thickness of the roughness zone of these samples becomes larger than $1 \mu \mathrm{m}$ (see Figure 1), which is often too much to exclude geometrical effects on the results of otherwise welldefined thermal exposures $[13,14,46]$.

A good compromise between sufficient optical absorption and sample roughness can be found for mean roughnesses $S_{\mathrm{q}}$ between $0.05 \mu \mathrm{m}$ and $0.20 \mu \mathrm{m}$. This corresponds to a mechanical treatment with 2500 grit grinding papers - a quite frequently, but not generally used preparation technique. Already small deviations of the surface roughness are sufficient to generate discrepancies of more than $100^{\circ} \mathrm{C}$ between actual and assumed sample temperature. Since the difference in the total energy consumption strongly depends on the actual sample roughness, these discrepancies become even more severe, when the reference sample possesses a very smooth (or very rough) surface. Again, a good compromise can be made by choosing a surface preparation with 2500 grit, which minimises the differences in relative energy absorption and hence in deviations of the sample temperature for a wide range of surface treatments. Finally, it should be stressed that the approach used here, while giving general guidelines towards sample preparation for high temperature corrosion experiments, relies on a number of assumptions. First, the temperature dependence of the heat capacities and all optical properties has been neglected. Second, the description of the sample's optical properties are not derived from a physical model [15], which implies that some assumptions, especially for the generalisation of the results to all wavelengths and angles of incidence are needed, which are not rigorously proven. Nevertheless, the main features of the approach used here are likely to represent the experimental situation sufficiently, especially during the initial stages of heating.

\section{ACKNOWLEDGEMENTS}

The authors thank Ms. Alexandra Vogel and Mr. Michael Adamek for helpful support with the sample preparation and the confocal laser scanning microscopy measurements. Financial support by the voestalpine Stahl GmbH (Austria) and the Christian Doppler Forschungsgesellschaft (Austria) is gratefully acknowledged.

\section{APPENDIX}

\section{A Model Parameters and Statistical Data for Reflectivity Modelling}

The optical properties of the multi-layered substrate were derived from ellipsometry data by using the Drude-Lorentz os- 
cillator model [7, 29, 30],

$$
\begin{aligned}
\epsilon_{r}(\omega) & =\epsilon^{\text {free }}(\omega)+\epsilon^{\text {bound }}(\omega) \\
& =\epsilon_{\infty}-\frac{\omega_{\mathrm{p}}^{2}}{\omega^{2}-i \omega \omega_{\tau}}+\sum_{j=0}^{k} \frac{\Omega_{\mathrm{p}, j}^{2}}{\Omega_{\mathrm{O}, j}^{2}-\omega^{2}+i \omega \Omega_{\tau, j}} .
\end{aligned}
$$

The meaning of the symbols is given in Table 3. Values, obtained from a least square fit, can be found in Table 4 .

\begin{tabular}{ll}
\hline$\omega$ & frequency \\
$\Omega_{\mathrm{O}, j}$ & resonance frequency of oscillator $j$ \\
$\epsilon_{r}(\omega)$ & dielectric function \\
$\Omega_{\mathrm{p}, j}$ & plasma frequency of oscillator $j$ \\
$\omega_{\mathrm{p}}$ & plasma frequency (free electron contribution) \\
$\Omega_{\tau, j}$ & damping constant of oscillator $j$ \\
$\omega_{\tau}$ & damping term (free electron contribution) \\
$i=\sqrt{-1}$ &
\end{tabular}

\begin{tabular}{|c|c|c|c|}
\hline \multicolumn{2}{|c|}{ oxide layer } & & \\
\hline$d_{1}$ & $1.20 \mathrm{~nm}$ & & \\
\hline$\epsilon(\infty)$ & 4.61 & & \\
\hline$\Omega_{\mathrm{O}, 0}$ & $37640 \mathrm{~cm}^{-1}$ & & \\
\hline$\Omega_{p, 0}$ & $29630 \mathrm{~cm}^{-1}$ & & \\
\hline$\Omega_{\tau, 0}$ & $9350 \mathrm{~cm}^{-1}$ & & \\
\hline \multicolumn{4}{|c|}{ substrate $(\mathrm{Fe}, 1 w t-\% \mathrm{Cr})$} \\
\hline$\epsilon(\infty)$ & 1 & & \\
\hline$\omega_{\mathrm{p}}$ & $43330 \mathrm{~cm}^{-1}$ & $\omega_{\tau}$ & $1797 \mathrm{~cm}^{-1}$ \\
\hline & $\Omega_{\mathrm{O}, j} / \mathrm{cm}^{-1}$ & $\Omega_{\mathrm{p}, j} / \mathrm{cm}^{-1}$ & $\Omega_{\tau, j} / \mathrm{cm}^{-1}$ \\
\hline$j=0$ & 64910 & 86780 & 37780 \\
\hline$j=1$ & 19830 & 50280 & 16590 \\
\hline$j=2$ & 9760 & 74140 & 24820 \\
\hline$j=3$ & 35140 & 15370 & 13120 \\
\hline
\end{tabular}

TABLE 3 Meaning of the symbols introduced in Eq. (7).

TABLE 4 Non-zero Drude-Lorentz model parameters used to model ellipsometry data of the Fe, $1 \mathrm{wt}-\% \mathrm{Cr}$ reference. Values were obtained from a least squares fit of the experimental data.

\section{B Theoretical Approach to Calculate Optical Heating Efficiency}

Following basic physical principles, the sample was assumed to be of a point-like geometry inside a hollow cylinder representing the furnace (Figure 7(a)). The cylinder further possesses the energy flux emission spectrum $E(\lambda)$ of an ideal black body at $1600^{\circ} \mathrm{C}\left(\lambda_{\max }=1.55 \mu \mathrm{m}\right)$. The power of the cylinder should be homogeneous across the entire surface and follows the behaviour of Planck's formula [47],

$$
E(\lambda)=\frac{2 \pi h c^{2}}{\lambda^{5}} \frac{1}{\exp \left(\frac{h c}{\lambda k T_{\mathrm{B}}}\right)-1} .
$$

The meaning of the symbols is given in Table 5 .

It needs to be emphasised that the total emitted energy of the cylinder surface remains constant which causes a decrease in
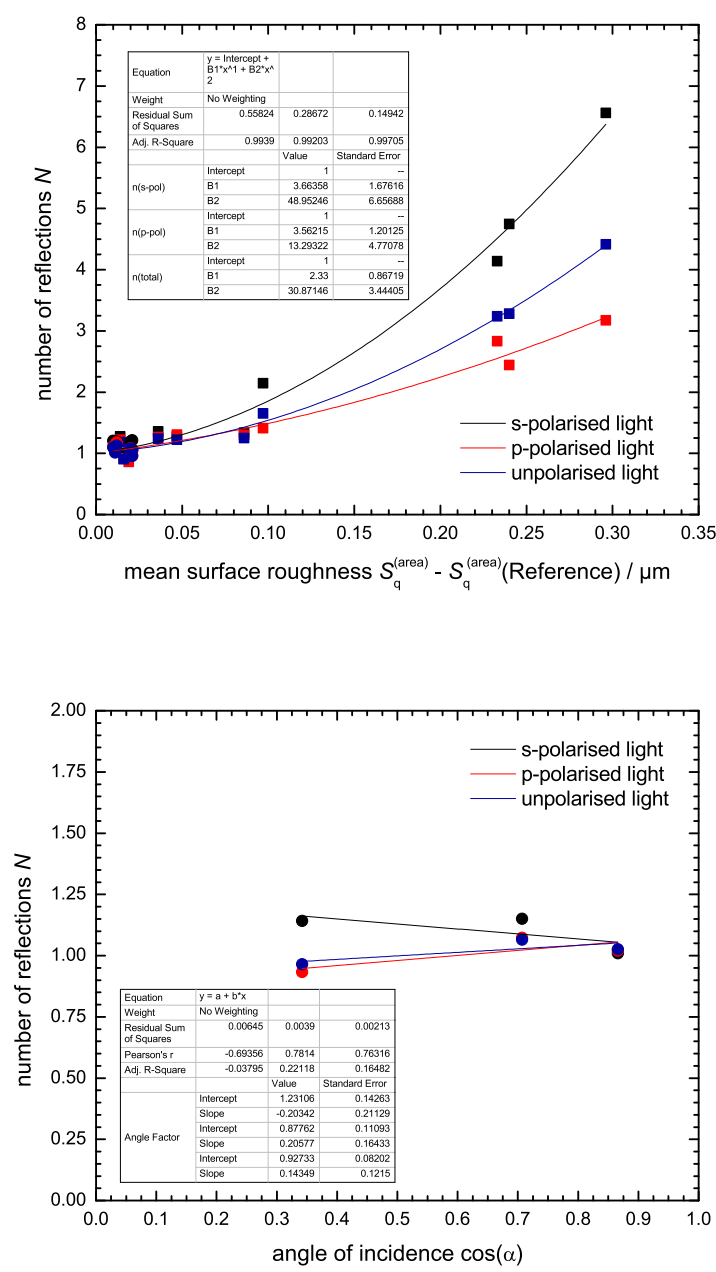

FIG. 9 Number of reflection events $N$, light $(\lambda=532 \mathrm{~nm})$ undergoes before being scattered into the far field on $\mathrm{Fe}, 1 \mathrm{wt}-\% \mathrm{Cr}$ surfaces depending on surface roughness at $\alpha=45^{\circ}$ (a). See Eqs. (2) and (3) for definitions. Angular dependency of $(R+S)$ for a surface polished with $1 \mu \mathrm{m}$ suspension with $S_{\mathrm{q}}^{(\text {area })}=0.026 \mu \mathrm{m}$, (b). In both sub-figures, the solid lines were derived from a fit function for both, ground samples

$(\boldsymbol{\square})$ and polished samples $(\bullet)$.

intensity, when moving away from the cylinder surface. It is further assumed that each point on the cylinder surface acts like a hemispherical emitter and the emitted light will not be reflected from the cylinder surface. Hence the overall energy flux $E_{\mathrm{smp}}$ that reaches the sample surface follows from

$$
E_{\mathrm{smp}}(\lambda)=E(\lambda) \frac{A_{\mathrm{smp}}}{2 \pi d^{2}}=E(\lambda) \frac{A_{\mathrm{smp}}}{2 \pi(r \cos \alpha)^{2}},
$$

where $d$ is the distance between emitter and sample and $r$ the cylinder's radius (see Figure 7(a)). The absorbed energy, depending on the wavelength and the angle of incidence can then be calculated by

$$
\dot{Q}_{\mathrm{smp}}=\iint E_{\mathrm{smp}}(\lambda)\left(1-R(\lambda, \alpha)^{N\left(S_{q}, \alpha\right)}\right) \pi r \mathrm{~d} \lambda \mathrm{d} l .
$$

By using the connection between the length of the cylinder and the angle of incidence,

$$
l=r \tan (\alpha) \longrightarrow \mathrm{d} l=\frac{r}{\cos ^{2}(\alpha)} \mathrm{d} \alpha .
$$




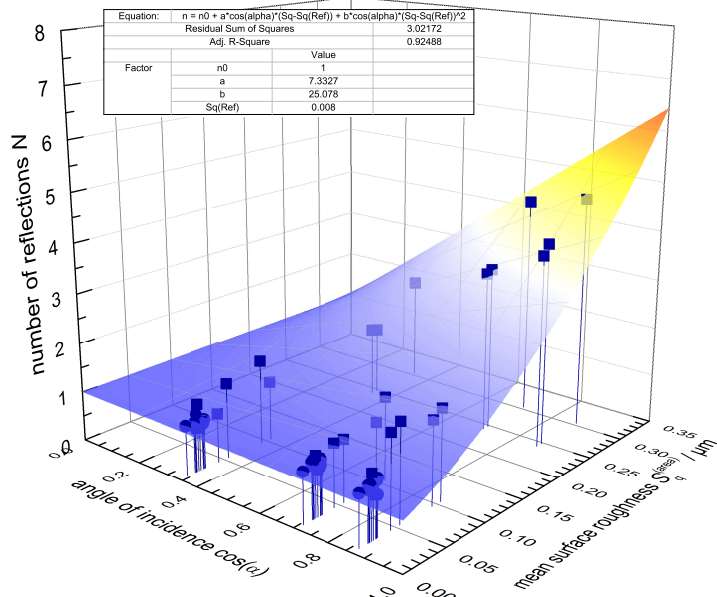

FIG. 10 Number of reflection events $N$ of light with $\lambda=532 \mathrm{~nm}$ on the surface of Fe, $1 \mathrm{wt}-\% \mathrm{Cr}$ alloys, depending on the mean surface roughness and the angle of incidence. The measurements were obtained from ground samples $(\boldsymbol{\square})$ and polished samples $(\bullet)$.

\begin{tabular}{ll}
\hline$E(\lambda)$ & energy flux emission spectrum of the furnace \\
$T_{\mathrm{B}}$ & black body temperature (example here: \\
& $\left.T_{\mathrm{B}}=1873 \mathrm{~K}\right)$ \\
$h$ & Planck's constant \\
$c$ & speed of light \\
$k$ & Boltzmann's constant \\
\hline
\end{tabular}

TABLE 5 Meaning of symbols in Eq. (8)

the implicit relations in the integral variables can then be reduced to only $\lambda$ and $\alpha$,

$$
\dot{Q}_{\mathrm{smp}}=\frac{A_{\mathrm{smp}}}{2} \iint E(\lambda)\left(1-R(\lambda, \alpha)^{N\left(S_{q}, \alpha\right)}\right) \mathrm{d} \lambda \mathrm{d} \alpha .
$$

Here, the upper boundary for the angle of incidence $\alpha_{\max }$ is defined by the total length of the furnace.

Results of the energy absorption, depending on the sample roughness and the length of the furnace are shown in Figure 7(b). The efficiency $\eta$ for optical heating of a sample then follows from its absorption properties, which is maximum at $R+S=0$ (see Eq. (1)),

$$
\eta=\frac{\iint E(\lambda)\left(1-R(\lambda, \alpha)^{N\left(S_{q}, \alpha\right)}\right) \mathrm{d} \lambda \mathrm{d} \alpha}{\iint E(\lambda) \mathrm{d} \lambda \mathrm{d} \alpha} .
$$

Similarly to the increase of the total energy input with higher $S_{\mathrm{q}}$, Figure 11 shows a decrease of heating efficiency for smoother surfaces (smaller $S_{\mathrm{q}}$ ). This originates from the higher $(R+S)$ of the samples. Although poorly visible in the graph, the calculations indicate a slight decrease of the heating efficiency with increasing cylinder length (higher $\left.\alpha_{\max }\right)$, which can again be attributed to changes of $(R+S)$ with $\alpha$.

\section{Temperature Difference during a Heating Cycle}

The energy conservation for a sample in an optical furnace is described by Eqs. (4) and (5). To obtain a multi-purpose

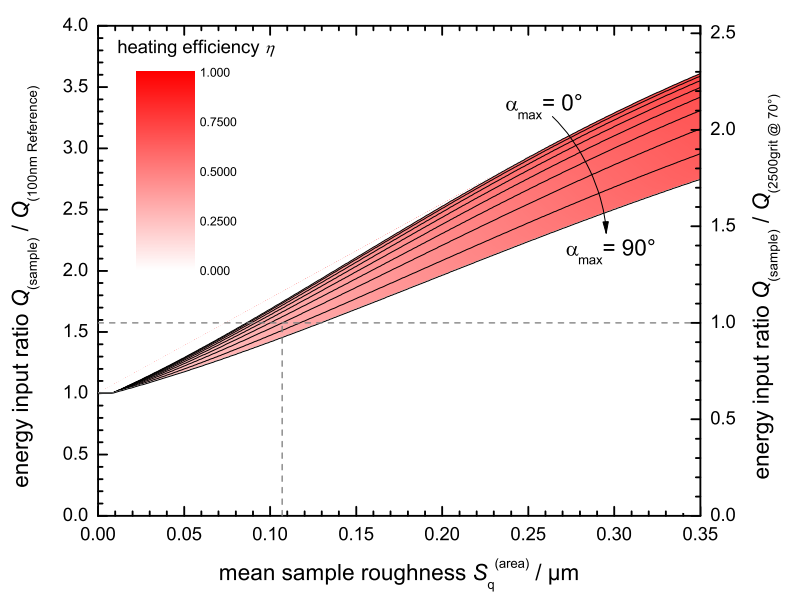

FIG. 11 Calculated optical heating efficiency $\eta$ of Fe, 1 wt- $\% \mathrm{Cr}$ alloys, depending on the sample roughness and energy input ratio for different maximum angles of incidence (defines the length of a homogeneous radiating cylinder). Note the different scales on both sides of the graph.

solution for the actual sample temperature $\tilde{T}$, by applying a defined heating programme to a reference $T$, one should first solve the energy input curve for Eq. (4). Second, the energy input for the reference will be multiplied with the energy input ratio for the sample and inserted into Eq. (5).

Since the full derivation of even the simplified case for Eq. (4) is a very complicated and lengthy procedure, the solution will be outlined by neglecting the influence of radiation and considering only the situation for sample and gas heating. The numerical result for the more general solution of Eqs. (4) and (5), including a radiation term, will be derived by the explicit Euler method and presented graphically.

The homogeneous solution of the ordinary differential equation

$$
m_{\mathrm{Fe}} c_{\mathrm{p}, \mathrm{Fe}} \frac{\mathrm{d} T}{\mathrm{~d} t}+\dot{n}_{\mathrm{gas}} c_{\mathrm{p}, \mathrm{gas}}\left(T-T_{\mathrm{in}}\right)=\dot{Q}_{\mathrm{ref}} .
$$

for the reference temperature can be obtained by setting its left-hand side to zero,

$$
\frac{\mathrm{d} T}{\mathrm{~d} t}+\frac{T}{\tau}=0
$$

where

$$
\tau^{-1} \equiv \frac{\dot{n}_{\mathrm{gas}} c_{\mathrm{p}, \mathrm{gas}}}{m_{\mathrm{Fe}} c_{\mathrm{p}, \mathrm{Fe}}}
$$

Solving Eq. (15) yields

$$
T_{(t)}=z \mathrm{e}^{-t / \tau} \quad(z \in \mathbb{R})
$$

The particular solution for the inhomogeneous case can be derived by applying the method of the variation of the constant [41]. This means that the integration constant $z$ from the homogeneous solution is now considered a function of time $t$, thus carrying the inhomogeneous term from Eq. (14) (righthand side). Considering these settings, one can calculate the first derivative for the reference temperature $T$ as

$$
\frac{\mathrm{d} T}{\mathrm{~d} t}=\dot{z}_{(t)} \mathrm{e}^{-t / \tau}-\frac{z_{(t)} \mathrm{e}^{-t / \tau}}{\tau}
$$


After inserting Eqs. (17) and (18) into Eq. (14) and simplifying terms, one obtains

$$
\dot{z}_{(t)}=\left(\dot{Q}_{\mathrm{ref},(t)}+\frac{T_{\mathrm{in}}}{\tau}\right) \mathrm{e}^{t / \tau}
$$

which is integrated to

$$
z_{(t)}=T_{\mathrm{in}} \mathrm{e}^{t / \tau}+\int_{0}^{t} \dot{Q}_{\mathrm{ref},(t)} e^{t / \tau} \mathrm{d} t+y \quad(y \in \mathbb{R}) .
$$

Finally, the solution for the reference temperature by applying an arbitrary power input $\dot{Q}_{(t)}$ can be expressed as

$$
\begin{aligned}
T_{(t)} & =z_{(t)} \mathrm{e}^{-t / \tau} \\
& =T_{\text {in }}+\mathrm{e}^{-t / \tau} \int_{0}^{t} \dot{Q}_{\text {ref, }(t)} \mathrm{e}^{t / \tau} \mathrm{d} t+y \mathrm{e}^{-t / \tau} \quad(y \in \mathbb{R}) .
\end{aligned}
$$

Inserting the initial conditions for the differential equation (both set-temperature of the reference and actual sample temperature at $t=0$ should be $T_{0}$ ), one obtains after simplification of terms,

$$
T_{(t)}=T_{0}+T_{\text {in }}\left(1-\mathrm{e}^{-t / \tau}\right)+\mathrm{e}^{-t / \tau} \int_{0}^{t} \dot{Q}_{\mathrm{ref},(t)} \mathrm{e}^{t / \tau} \mathrm{d} t
$$

A similar expression is obtained for the actual sample temperature $\tilde{T}$ as

$$
\tilde{T}_{(t)}=T_{0}+T_{\text {in }}\left(1-\mathrm{e}^{-t / \tau}\right)+\mathrm{e}^{-t / \tau} \int_{0}^{t} \dot{Q}_{\mathrm{smp},(t)} \mathrm{e}^{t / \tau} \mathrm{d} t .
$$

The effect of the set-temperature of the reference to the actual temperature of the sample can be derived by correlating the obtained results, considering the energy input into the sample as multiple of the energy input into the reference (see Eq. (5)) one can combine Eqs. (22) and (23) into

$$
\begin{aligned}
\tilde{T}_{(t)}= & T_{0} \mathrm{e}^{-t / \tau}+T_{\text {in }}\left(1-\mathrm{e}^{-t / \tau}\right) \\
& +f\left(T_{(t)}-T_{0} \mathrm{e}^{-t / \tau}-T_{\text {in }}\left(1-\mathrm{e}^{-t / \tau}\right)\right),
\end{aligned}
$$

which simplifies to

$$
\tilde{T}_{(t)}=f T_{(t)}+T_{0}(1-f) \mathrm{e}^{-t / \tau}+T_{\text {in }}(1-f)\left(1-\mathrm{e}^{-t / \tau}\right) .
$$

The more general case including energy loss by radiation can not be derived by the same method. Due to an increased complexity of the radiation term, an explicit solution, containing the time-dependent energy input $\dot{Q}_{\text {ref, }(t)}$ can not be given. Hence numerical solutions will be provided, which were derived from Eq. (5) when the energy input is known, or by using

$$
\begin{aligned}
\frac{\mathrm{d} \tilde{T}_{(t)}}{\mathrm{d} t}= & f \frac{\mathrm{d} T_{(t)}}{\mathrm{d} t}+\frac{1}{\tau}\left(f T_{(t)}-\tilde{T}_{(t)}\right)+\frac{1-f}{\tau} T_{\mathrm{in}} \\
& +\frac{\epsilon \sigma A_{\mathrm{smp}}}{m_{\mathrm{Fe}} c_{\mathrm{p}, \mathrm{Fe}}}\left(f T_{(t)}^{4}-\tilde{T}_{(t)}^{4}\right) .
\end{aligned}
$$

for the deviation from an arbitrary (but known) settemperature programme.

To give a graphical representation of obtained results, two different cases of sample heating will be considered. Figure 12
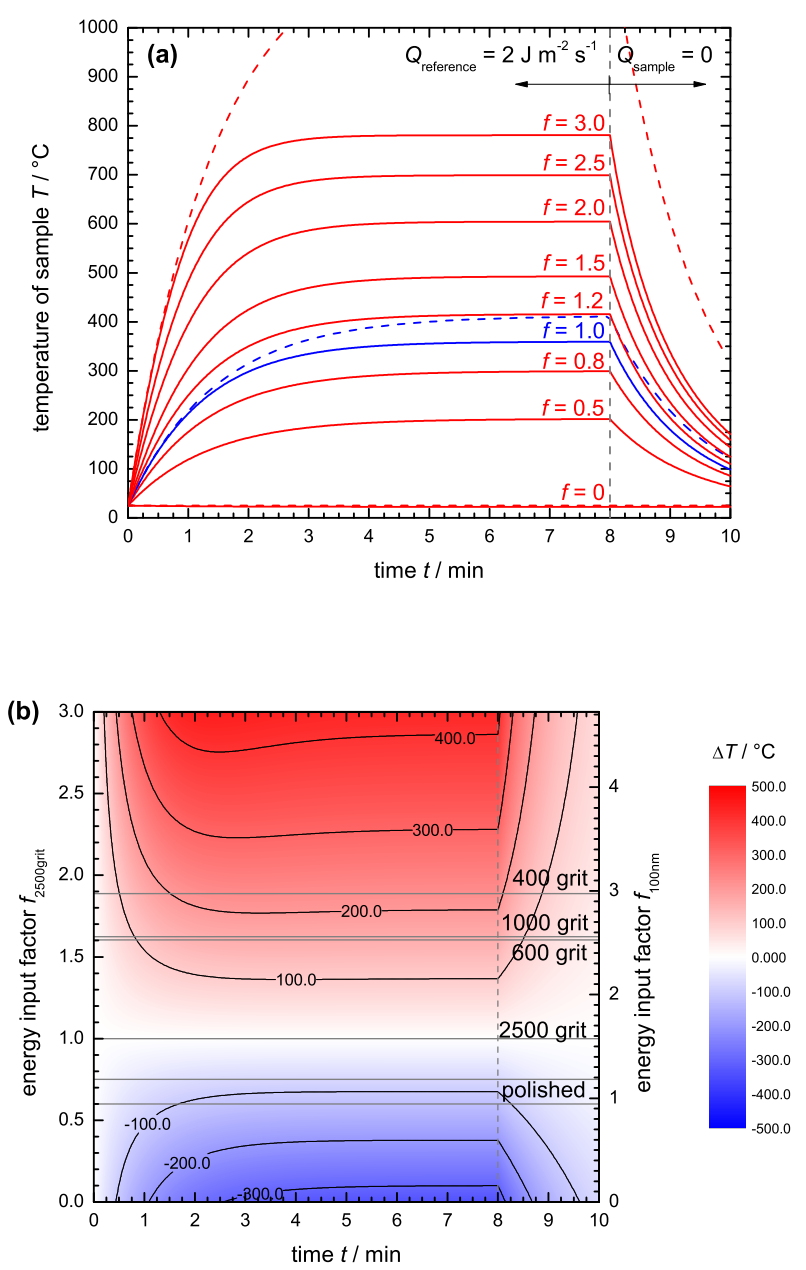

FIG. 12 Calculated temperature evolution in Fe, 1 wt- $\% \mathrm{Cr}$ samples with different surface roughness, by exposing the same to a constant optical energy $\dot{Q}_{\text {ref }}=2 \mathrm{~J} \mathrm{~m}^{-2} \mathrm{~s}^{-1}$. Dashed lines in (a) indicate the analytical solution of the temperature without thermal radiation. All simulations were calculated for different energy input ratios $f$ and a furnace with $\alpha_{\max }=70^{\circ}$.

depicts the actual sample temperature by using a constant optical input intensity $\dot{Q}_{\text {ref }}$ of the furnace. The situation without radiation losses can be solved quite easily by employing Eq. (22) for the actual temperature of the reference material and inserting these results into Eq. (23). Both, the actual temperature as well as the temperature difference between sample and reference are shown. As can be seen, heating rates and final temperature strongly depend on the surface roughness. A close look at the temperature deviation in Figure 12(b) reveals that the plot is not symmetrical around the set-temperature programme $(f=1)$ and the hot zone values are slightly smaller than the under-cooled area. This effect originates from the energy loss due to radiation.

A case closer to many laboratory environments, as shown in Figure 13, compares the actual sample temperature by applying a commonly used set-temperature programme to a reference. Again, severe differences in heating rate and the isothermal temperature section can be seen. Obtained temperatures may differ by more than $100^{\circ} \mathrm{C}$ if only minor differences of the roughness are present. 

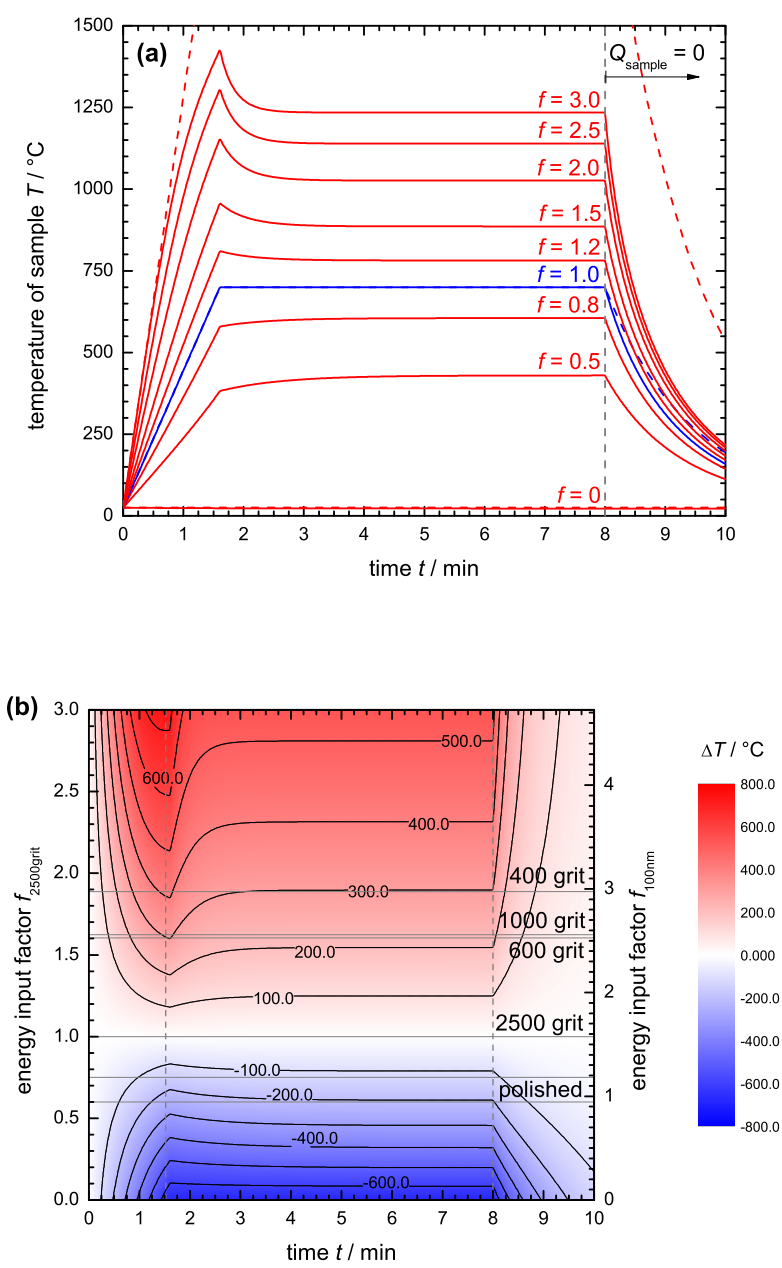

FIG. 13 Calculated temperature evolution of optically heated $\mathrm{Fe}$, $1 \mathrm{wt}-\% \mathrm{Cr}$ samples with different surface roughness (a) and temperature deviation for different energy input ratios (b). The set-temperature programme for a sample, ground with 2500 grit $\left(S_{\mathrm{q}}^{\text {(area })}=0.107 \mu \mathrm{m}\right)$ has been composed of linear heating with $7 \mathrm{~K} \mathrm{~s}^{-1}$ up to $700^{\circ} \mathrm{C}$, isothermal dwelling for $6.5 \mathrm{~min}$ and cooling in the flowing gas stream. Dashed lines in (a) indicate the analytical solution of the temperature without heat radiation. All simulations were calculated for different energy input ratios $f$ and a furnace with $\alpha_{\max }=70^{\circ}$

\section{References}

[1] W. J. Quadakkers, J. Żurek, and M. Hänsel, "Effect of Water Vapor on High-temperature Oxidation of FeCr Alloys," JOM 61, 44-50 (2009).

[2] H. Yin, S. L. I. Chan, W. Y. D. Yuen, and D. J. Young, "Temperature Effects on the 0xidation of Low Carbon Steel in $\mathrm{N}_{2}-\mathrm{H}_{2}-\mathrm{H}_{2} \mathrm{O}$ at $800-1200^{\circ} \mathrm{C}$," 0xid. Met. 77, 305-323 (2012).

[3] J. Kalivodova, D. Baxter, M. Schütze, and V. Rohr, "Gaseous corrosion of alloys and novel coatings in simulated environments for coal, waste and biomass boilers," Mater. Corros. 56, 882-889 (2005).

[4] J. Warris, M. Suleman, F. Mahmood, and H. Ahmed, "Kinetics of the formation of cobalt disilicide at high temperature under rapid electron beam heating," J. Mater. Sci. Lett. 13, 96-98 (1994).

[5] D. Leong, M. Harry, K. J. Reeson, and K. P. Homewood, "A silicon/iron-disilicide light-emitting diode operating at a wavelength of $1.5 \mu \mathrm{m}, "$ Nature $387,686-688$ (1997).
[6] S. R. Levine, E. J. Opila, M. C. Halbig, J. D. Kiser, M. Singh, and J. A. Salem, "Evaluation of ultra-high temperature ceramics for aeropropulsion use," J. Eur. Ceram. Soc. 22, 2757-2767 (2002).

[7] C. F. Bohren, and D. R. Huffman, Absorption and Scattering of Light by Small Particles ( $1^{\text {st }}$ edition, Wiley-VCH, Weinheim, 2008).

[8] A. Roos, M. Bergkvist, and C. G. Ribbing, "Optical Scattering from Oxidized Metals. 1: Model Formulation and Properties," Appl. Optics 28, 1360-1364 (1989).

[9] A. Roos, M. Bergkvist, and C. G. Ribbing, "Optical Scattering from Oxidized Metals. 1: Model Formulation and Properties; Errata," Appl. Optics 28, 3795_1 (1989).

[10] M. Bergkvist, A. Roos, C. G. Ribbing, J. M. Bennett, and L. Mattson, "Optical Scattering from 0xidized Metals. 2: Model Verification for Oxidized Copper," Appl. Optics 28, 3902-3907 (1989).

[11] T. A. Cermer, "Measurement of Roughness of Two Interfaces of a Dielectric Film by Scattering Ellipsometry," Phys. Rev. Lett. 85, 349-352 (2000).

[12] M. Karamemehdović, P. E. Hansen, and T. Wriedt, "An efficient rough-interface scattering model for embedded nano-structures," Thin Solid Films 541, 51-56 (2013).

[13] M. Auinger, D. Vogel, A. Vogel, M. Spiegel, and M. Rohwerder, "A novel laboratory set-up for investigating surface and interface reactions during short term annealing cycles at high temperatures," Rev. Sci. Instr. 84, 085108 (2013).

[14] L. Niewolak, M. Malessa, S. Y. Coleman, W. J. Quadakkers, and M. Schütze, "Influence of cycling parameter variation on thermal cyclic oxidation testing of high temperature materials (COTEST)," Mater. Corros. 57, 31-42 (2006).

[15] Light Scattering and Nanoscale Surface Roughness, A. A. Maradudin eds., ( $1^{\text {st }}$ edition, Springer, New York, 2007).

[16] S. Schröder, A. Duparré, L. Coriand, A. Tünnermann, D. H. Penalver, and J. E. Harvey, "Modeling of light scattering in different regimes of surface roughness," Opt. Express 19, 9820-9835 (2011).

[17] T. A. Germer, "Polarized light scattering by microroughness and small defects in dielectric layers," J. Opt. Soc. Am. A 18, 1279-1288 (2001).

[18] G. Vasan, Y. Chen, and A. Erbe, "Computation of Surface-Enhanced Infrared Absorption Spectra of Particles at a Surface through the Finite Element Method," J. Phys. Chem. C 115, 3025-3033 (2011).

[19] G. Vasan, and A. Erbe, "Incidence angle dependence of the enhancement factor in attenuated total reflection surface enhanced infrared absorption spectroscopy studied by numerical solution of the vectorial Maxwell equations," Phys. Chem. Chem. Phys. 14, 14702-14709 (2012).

[20] A. G. Skirtach, D. G. Kurth, and H. Möhwald, "Laser-embossing nanoparticles into a polymeric film," Appl. Phys. Lett. 94, 093106 (2009).

[21] J. H. Kim, S. H. Ehrman, G. W. Mulholland, and T. A. Germer, "Polarized light scattering by dielectric and metallic spheres on oxidized silicon surfaces," Appl. Optics 43, 585-591 (2004).

[22] S.-H. Hsu, Y.-C. Chang, Y.-C. Chen, P.-K. Wei, and Y. D. Kim, "Optical metrology of randomly-distributed Au colloids on a multilayer film," Opt. Express 18, 1310-1315 (2010).

[23] B. C. Bergner, T. A. Germer, and T. J. Suleski, "Effective medium approximations for modeling optical reflectance from gratings with rough edges," J. Opt. Soc. Am. A 27, 1083-1090 (2010).

[24] E. Marx, T. R. Lettieri, and T. V. Vorburger, "Light scattering by sinusoidal surfaces: illumination windows and harmonics in stan- 
dards," Appl. Optics 34, 1269-1277 (1995).

[25] P. G. Appleyard, "Modelled infrared extinction and attenuation performance of atmospherically disseminated high aspect ratio metal nanoparticles," J. Opt. A: Pure Appl. Opt. 9, 278-300 (2007).

[26] M. Sun, Y. Fang, Z. Yang, and H. Xu, "Chemical and electromagnetic mechanisms of tip-enhanced Raman scattering," Phys. Chem. Chem. Phys. 11, 9412-9419 (2009).

[27] G. Petzow, V. Carle, and U. Harnisch, Metallographic etching (2 ${ }^{\text {nd }}$ edition, Materials Park: ASM International, Ohio, 1999).

[28] T. C. Choy, Effective Medium Theory - Principles and Applications (Clarendon Press, 0xford, 1999).

[29] M. Born, and E. Wolf, Principles of Optics ( $7^{\text {th }}$ edition, Cambridge University Press, Cambridge, 2002).

[30] G. R. Fowles, Introduction to Modern Optics ( $2^{\text {nd }}$ edition, Dover Publications, New York, 1989).

[31] E. Hecht, Optics (4 ${ }^{\text {th }}$ edition, Addison Wesley, San Francisco, 2002).

[32] B. J. Griffiths, R. H. Middleton, and B. A. Wilkie, "Light scattering for the measurement of surface finish: a review," Int. J. Prod. Res. 32, 2683-2694 (1994).

[33] D. E. Aspnes, J. B. Theeten, and F. Hottier, "Investigation of effective-medium models of microscopic surface roughness by spectroscopic ellipsometry," Phys. Rev. B 20, 3292-3302 (1979).

[34] T. A. Germer, and C. C. Asmail, "Polarization of light scattered by microrough surfaces and subsurface defects," J. Opt. Soc. Am. A 16, 1326-1332 (1999).

[35] J. C. Stover, Optical Scattering: Measurement and Analysis ( $2^{\text {nd }}$ edition, SPIE Press, Bellingham, 1995).

[36] R. J. Dick, K. J. Heater, V. D. McGinniss, W. F. McDonald, and R. E. Russel, "Comparison of the Effectiveness of Electric IR and Other Energy Sources To Cure Powder Coatings," J. Coating. Technol. 66, 23-38 (1994).
[37] S. Mokkapati, and C. Jagadish, "III-V compound SC for optoelectronic devices," Mater. Today 12, 22-32 (2009).

[38] J. R. Howell, R. Siegel, and M. P. Menguc, Thermal Radiation Heat Transfer ( $5^{\text {th }}$ edition, CRC Press, Boca Raton, 2010).

[39] M. Lax, "Temperature rise induced by a laser beam," J. Appl. Phys. 48, 3919-3924 (1977).

[40] J. P. Traverse, P. Fort, H. Ganda, and R. Saporte, "Investigation of optical properties of iron, chromium, and nickel oxide based coatings," Sol. Energ. Mat. Sol. C. 28, 195-207 (1992).

[41] D. W. Jordan, and P. Smith, Nonlinear Ordinary Differential Equations - An Introduction for Scientists and Engineers ( $4^{\text {th }}$ edition, Oxford University Press, 0xford, 2007).

[42] D. R. Lide, CRC Handbook of Chemistry and Physics (CRC Press, Boca Raton, 2010).

[43] L. del Campo, R. B. Pérez- Saéz, X. Esquisabel, I. Fernández, and M. J. Tello, "New experimental device for infrared spectral directional emissivity measurements in a controlled environment," Rev. Sci. Instrum. 77, 113111 (2006).

[44] S. G. Gopalakrishnan, P. Huczkowski, J. Pernpeintner, T. Fend, H. Hattendorf, R. Iskandar, J. Mayer, et al., “Composition modifications and heat treatment procedures for increasing the emissivity of alumina surface scales on FeCrAl alloys," Mater. High Temp. 29, 249-256 (2012).

[45] M. Nowak, "Determination of optical constants and average thickness of inomogeneous-rough thin films using spectral dependence of optical transmittance," Thin Solid Films 254, 200-210 (1995).

[46] C. Mitterer, F. Holler, F. Üstel, and D. Heim, "Application of hard coatings in aluminium die casting - soldering, erosion and thermal fatigue behaviour," Surf. Coat. Tech. 125, 233-239 (2000).

[47] R. Kingston, Optical Sources, Detectors, and Systems - Fundamentals and Applications ( $1^{\text {st }}$ edition, Academic Press, Boston, 1995). 